\title{
A!
}

This is an electronic reprint of the original article.

This reprint may differ from the original in pagination and typographic detail.

Gasparinetti, S.; Solinas, P.; Pugnetti, S.; Fazio, Rosario; Pekola, J.P.

\section{Environment-Governed Dynamics in Driven Quantum Systems}

Published in:

Physical Review Letters

DOI:

10.1103/PhysRevLett.110.150403

Published: 12/04/2013

Document Version

Publisher's PDF, also known as Version of record

Please cite the original version:

Gasparinetti, S., Solinas, P., Pugnetti, S., Fazio, R., \& Pekola, J. P. (2013). Environment-Governed Dynamics in Driven Quantum Systems. Physical Review Letters, 110(15), 1-5. [150403].

https://doi.org/10.1103/PhysRevLett.110.150403

This material is protected by copyright and other intellectual property rights, and duplication or sale of all or part of any of the repository collections is not permitted, except that material may be duplicated by you for your research use or educational purposes in electronic or print form. You must obtain permission for any other use. Electronic or print copies may not be offered, whether for sale or otherwise to anyone who is not an authorised user. 


\title{
Environment-Governed Dynamics in Driven Quantum Systems
}

\author{
S. Gasparinetti, ${ }^{1, *}$ P. Solinas, ${ }^{1,2}$ S. Pugnetti, ${ }^{3}$ R. Fazio, ${ }^{3}$ and J. P. Pekola ${ }^{1}$ \\ ${ }^{1}$ Low Temperature Laboratory (OVLL), Aalto University, P.O. Box 15100, FI-00076 Aalto, Finland \\ ${ }^{2}$ COMP Centre of Excellence, Department of Applied Physics, Aalto University School of Science, \\ P.O. Box 11000, 00076 Aalto, Finland \\ ${ }^{3}$ NEST, Scuola Normale Superiore and Istituto Nanoscienze-CNR, I-56126 Pisa, Italy
}

(Received 13 December 2012; published 12 April 2013)

\begin{abstract}
We show that the dynamics of a driven quantum system weakly coupled to the environment can exhibit two distinct regimes. While the relaxation basis is usually determined by the system + drive Hamiltonian (system-governed dynamics), we find that under certain conditions it is determined by specific features of the environment, such as, the form of the coupling operator (environment-governed dynamics). We provide an effective coupling parameter describing the transition between the two regimes and discuss how to observe the transition in a superconducting charge pump.
\end{abstract}

PACS numbers: 03.65.Yz, 03.67.Lx, 42.50.Hz, 85.25.Cp

Introduction.-Understanding how quantum systems interact with the environment [1] is of paramount importance in quantum information science. While unveiling how the classical world emerges from the quantum one [2], it can also lead to a better protection against decoherence effects on the way towards the realization of a quantum computer [3].

A standard approach to the dynamics of open quantum systems boils the problem down to the measurement of decoherence rates, distinguishing between coherence loss, or dephasing, and relaxation. While this approach has successfully described a variety of quantum systems, it only offers a limited insight into the dynamics of decoherence. A promising line of work developed in the last decade exploits the possibility of coupling the system to an engineered reservoir [4-12].

As new and more accurate ways are found of harnessing the dynamic evolution of quantum systems, it becomes increasingly important to understand how the interaction with the environment is affected by a time-dependent modulation of the system parameters. Indeed, the study of dissipation in driven quantum systems is a longestablished topic [13] that keeps finding new applications to quantum pumping [14-16], quantum computation $[17,18]$, and possibly even biological systems [19,20].

In this Letter, we consider a periodically driven quantum system in the presence of a weakly coupled environment. We show that under certain conditions decoherence takes place in a preferred basis determined by specific features of the environment, such as, the type of noise, rather than of the system. We label this unusual regime as environmentgoverned dynamics (EGD), as opposed to the more familiar system-governed dynamics (SGD). We introduce an effective coupling parameter that presides over the transition between SGD and EGD. This parameter can be tuned by changing the properties of the drive. Our analysis is general and applies to optical and solid-state systems alike.
As a relevant example, we propose to observe the transition in a superconducting charge pump [21,22]. In this system the transition is controlled by an accessible experimental parameter and can be explored by measuring the pumped charge.

Floquet-Born-Markov master equation.-We consider a quantum system whose unitary evolution is governed by a periodic Hamiltonian $H$, so that $H(t)=H(t+\tau)$, where $\tau$ is the period. According to the Floquet theorem, the Schrödinger equation admits solutions (Floquet states) of the form $\left|\Psi_{\alpha}(t)\right\rangle=e^{-i \epsilon_{\alpha} t / \hbar}\left|\varphi_{\alpha}(t)\right\rangle$, where the Floquet mode $\left|\varphi_{\alpha}(t)\right\rangle$ satisfies $\left|\varphi_{\alpha}(t+\tau)\right\rangle=\left|\varphi_{\alpha}(t)\right\rangle$ and $\epsilon_{\alpha}$ is its corresponding quasienergy. Quasienergies and their associated modes are defined up to the translation $\epsilon_{\alpha} \rightarrow \epsilon_{\alpha}+\hbar \Omega$, where $\Omega=2 \pi / \tau$. As such, all quasienergies can be mapped into the first Brillouin zone $\left[-\frac{1}{2} \hbar \Omega, \frac{1}{2} \hbar \Omega\right]$. The Hamiltonian describing the system and its environment is given by $H_{\mathrm{tot}}=H(t)+H_{E}+H_{S E}$, where $H_{E}$ describes the environmental degrees of freedom and $H_{S E}$ is the interaction term, that we assume of the form $H_{S E}=g A \otimes E$, where $g$ is an adimensional coupling constant and $A$ and $E$ are operators acting in the Hilbert space of the system and the environment, respectively.

The general procedure for deriving the master equation (ME) in the Floquet basis and in the Born-Markov approximation is outlined in Refs. [13,23]. The superoperator describing the time evolution of the density matrix is expressed as a series of time-independent coefficients multiplied by phase factors of the form $e^{i \Delta_{\alpha, \beta, k}-i \Delta_{\gamma, \delta, k^{\prime}}}$, with $\Delta_{\alpha, \beta, k}=\epsilon_{\alpha}-\epsilon_{\beta}+k \Omega$. Starting from this expression, we perform a partial secular approximation (PSA): we neglect all terms with $k \neq k^{\prime}$ while keeping those with $k=k^{\prime}$ also when $\epsilon_{\alpha} \neq \epsilon_{\beta}$. A residual time dependence due to terms oscillating like $e^{i\left(\epsilon_{\alpha}-\epsilon_{\beta}\right) t}$ disappears when passing from the basis of Floquet states to that of Floquet modes. Our result, written in the basis of Floquet modes and in the Schrödinger picture, reads: 


$$
\dot{\rho}_{\alpha \beta}=-i\left(\epsilon_{\alpha}-\epsilon_{\beta}\right) \rho_{\alpha \beta}+\sum_{\gamma, \delta} \rho_{\gamma, \delta} \mathcal{R}_{\alpha, \beta, \gamma, \delta}
$$

where

$$
\begin{aligned}
\mathcal{R}_{\alpha, \beta, \gamma, \delta}= & \Gamma^{+}{ }_{\alpha, \gamma, \beta, \delta}+\Gamma^{-}{ }_{\alpha, \gamma, \beta, \delta}-\delta_{\delta, \beta} \sum_{\mu} \Gamma^{+}{ }_{\mu, \gamma, \mu, \alpha} \\
& -\delta_{\gamma, \alpha} \sum_{\mu} \Gamma^{-}{ }_{\mu, \beta, \mu, \delta}
\end{aligned}
$$

and

$$
\begin{aligned}
\Gamma_{\alpha, \beta, \gamma, \delta}^{+} & =\sum_{k} S\left(\Delta_{\alpha, \beta, k}\right) X_{\alpha, \beta, k}\left(X_{\gamma, \delta, k}\right)^{*}, \\
\Gamma_{\alpha, \beta, \gamma, \delta}^{-} & =\sum_{k} S\left(\Delta_{\gamma, \delta, k}\right) X_{\alpha, \beta, k}\left(X_{\gamma, \beta, k}\right)^{*} .
\end{aligned}
$$

We have introduced the following quantities:

$$
\begin{aligned}
& S(\omega)=\theta(\omega) J(\omega) n_{\mathrm{th}}(\omega)+\theta(-\omega) J(-\omega)\left[1+n_{\mathrm{th}}(-\omega)\right], \\
& \Delta_{\alpha \beta k}=\epsilon_{\alpha}-\epsilon_{\beta}+k \Omega, \\
& X_{\alpha \beta k}=\int_{0}^{\tau} d t e^{-i k \Omega t}\left\langle\varphi_{\alpha}\left|A \varphi_{\beta}\right|\right\rangle,
\end{aligned}
$$

where $\theta(\omega)$ is the Heaviside function, $J(\omega)$ the spectral density of the bath, and $n_{\mathrm{th}}(\omega)$ is the Bose-Einstein distribution.

A few remarks are in order. First, Eq. (1) cannot be cast into a Pauli ME [24]; i.e., the equations for the diagonal and off-diagonal terms are still coupled. This is due to the fact that we performed a PSA instead of a full secular approximation; we expect the additional terms to become important close to degeneracies in the Floquet spectrum $[25,26]$. The PSA itself is justified provided the drive period is much faster than the decoherence time. This may not be true in the adiabatic limit, where other terms should instead be retained [27]. We have numerically checked the validity of the PSA in the present case by including more oscillating terms in the ME and comparing the results; the agreement is excellent.

Equation (1) is formally akin to that of an undriven system, with quasienergies playing the role of ordinary eigenenergies and effective rates given by Eq. (3). The presence of a drive manifests itself in the sum over $k$ in Eq. (3), allowing the system to exchange energy with the environment in any of the amounts $\Delta_{\alpha, \beta, k}$. The magnitude of this exchange is determined by the noise matrix elements $X_{\alpha, \beta, k}$, telling whether the Floquet modes possess those energies and to what extent the coupling operator $A$ allows the energy transfer to take place. Note that while each individual term in the sums (3) satisfies the detailed balance, the overall rates $\Gamma^{ \pm}$in general do not.

Environment-governed dynamics.-Equation (1) suggests that the dynamics of the coherences is determined by a competition between two terms. Let us now focus our attention on two states, $\alpha$ and $\beta$. For $\alpha \neq \beta$, the first term in Eq. (1) is the Floquet energy gap $\mathcal{E}=\epsilon_{\alpha}-\epsilon_{\beta}$, stemming from the nondissipative dynamics of the driven quantum system. The second term describes the effect of dissipation. For sufficiently small values of $\mathcal{E}$, that is, close to a degeneracy in the Floquet spectrum, the dissipative term can dominate in Eq. (1). As a result, the dynamics of states $\alpha, \beta$ is strongly affected by the environment even if the system and the environment are only weakly coupled, i.e., $g \ll 1$. This is due to the presence of a nearly resonant driving field introducing an energy scale, the Floquet gap, that can be much smaller than those of the undriven system.

Let us now estimate the magnitude of the rates $\Gamma^{ \pm}$in Eq. (3). We consider an Ohmic environment, whose spectral density is given by $J(\omega)=a \omega f_{c}\left(\omega / \omega_{c}\right)$, where $a$ is a dimensionless constant, $f_{c}$ a cutoff function, and $\omega_{c}$ the cutoff frequency. In the limit $\Omega \ll \omega_{c}$ our results are independent of $\omega_{c}$ and the explicit form of $f_{c}$. As the sum in Eq. (3) runs over $k$, we may expect the dominant contributions to come from high-frequency modes (large $k$ ) such that $X_{\alpha \beta k}$ does not vanish and $k \Omega<\omega_{c}$. These contributions are of order $g^{2}(\mathcal{E}+k \Omega) \approx k g^{2} \Omega$.

We define an effective coupling parameter as

$$
\chi=g^{2} \Omega / \mathcal{E} .
$$

If $\chi \ll 1$ we are in the SGD regime. This corresponds to a decoherence time much longer than the period of Rabi oscillations between Floquet modes. By contrast, when $\chi \gtrsim 1$ we enter the EGD regime. This regime is realized close to a (quasi)degeneracy of the Floquet spectrum, where Rabi oscillations between Floquet modes are slower than the decoherence time. We remark that the coupling parameter $\mathcal{X}$ is controlled by the Floquet gap $\mathcal{E}$, which can be tuned by changing the drive parameters. To appreciate the key role played by the drive, we observe that an undriven system with an arbitrarily small energy gap cannot exhibit EGD. This is due to the fact that for any Ohmic and super-Ohmic environment the transition rates vanish for a vanishing energy gap.

$S G D$ to EGD transition.-As the foregoing discussion highlights, a transition between SGD and EGD is to be expected every time (i) a degeneracy or quasidegeneracy is encountered in the Floquet spectrum, and (ii) the noise operator actively couples the Floquet states. These conditions are quite general and may be found in a variety of systems; even a simple quantum bit driven by a monochromatic drive can exhibit EGD. Furthermore, environments with a more structured density of states, such as those found in cavity quantum electrodynamics architectures, may offer additional insight into the EGD regime.

We now provide an explicit example of SGD to EGD transition in a driven two-level system. Using a pseudospin formalism, we write the system Hamiltonian as $H(t)=$ $\vec{\sigma} \cdot \vec{B}(t)$, where $\vec{\sigma}=\left\{\sigma_{x}, \sigma_{y}, \sigma_{z}\right\}$ are the Pauli operators and $\vec{B}(t)$ an effective magnetic field. We consider a drive that modulates $\vec{B}$ along the loop shown in Fig. 1(a). This drive is a realization of Landau-Zener-Stückelberg interference [28] 
(a)

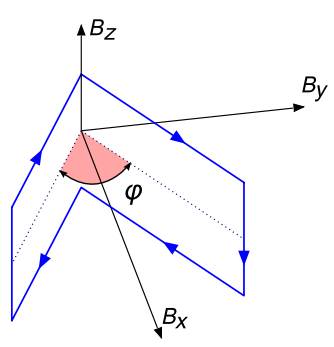

(b)

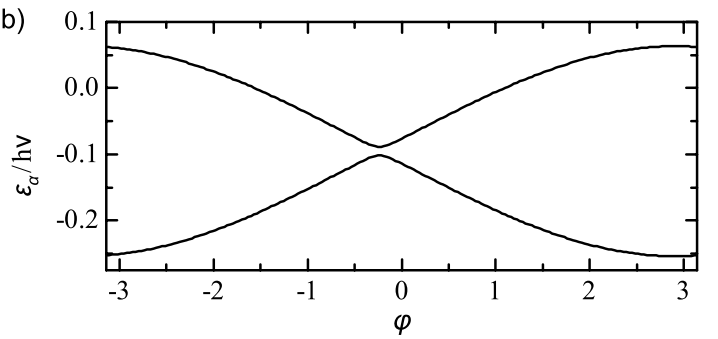

FIG. 1 (color online). A model driven two-level system. (a) Due to the driving field, the tip of the effective magnetic field $\vec{B}$ draws a closed loop in pseudospin space. The explicit time dependence of $\vec{B}$ can be obtained by that of the parameters $J_{L}, J_{R}$, and $n_{g}$, provided in Fig. 3(b), with the help of the expressions given in the text. The solid angle spanned by $\vec{B}$ is controlled by the angle $\varphi$, that we regard as a tunable parameter. (b) Quasienergy spectrum as a function of $\varphi$. A weakly avoided quasienergy crossing occurs at $\varphi_{c}=-0.26$.

with geometric phases [29]. Its geometric properties provide a convenient way of tailoring the Floquet spectrum. In particular, we study the effects of varying the angle $\varphi$ [see Fig. 1(a)], which determines the solid angle spanned by $\vec{B}$ in the pseudospin space during a drive period. In Fig. 1(b) we plot the quasienergy spectrum of the system versus $\varphi$. The plot is obtained by numerically solving the Schrödinger equation for the evolution operator generated by $H$. The quasienergy gap $\mathcal{E}$ sharply decreases near $\varphi=\varphi_{c}$, where a weakly avoided quasienergy crossing occurs. Close to $\varphi_{c}$, we thus expect EGD to be attained.

We characterize the transition from SGD to EGD by studying the quasistationary state approached by the driven system in the presence of dissipation. For the purpose of illustration, we consider a zero-temperature environment [30]. We write the quasistationary density matrix $\rho_{\text {st }}$ as $\rho_{\text {st }}=1 / 2(\mathbb{1}+\vec{n} \cdot \vec{\Sigma})$ where $\vec{\Sigma}$ is the vector of Pauli operators in the Floquet-mode basis. In this way, the residual coherence between Floquet states is associated to the quantity $n_{\perp}=\sqrt{n_{x}^{2}+n_{y}^{2}}$.

In Fig. 2(a) we plot $n_{z}$ (full line) and $n_{\perp}$ (dashed line) versus $\varphi$ in a neighborhood of $\varphi_{c}$. We choose the noise operator $A=\sigma_{z}$ and $g^{2}=0.01$. As $\varphi$ approaches $\varphi_{c}$ from either side, we witness the transition from SGD to EGD, signalled by a revival of the coherence between Floquet states. This revival is the first distinctive feature of EGD. We then fix $\varphi$ to a value close to $\varphi_{c}$ and change $\chi$ by changing $g^{2}$, to which $\chi$ is proportional. The results are
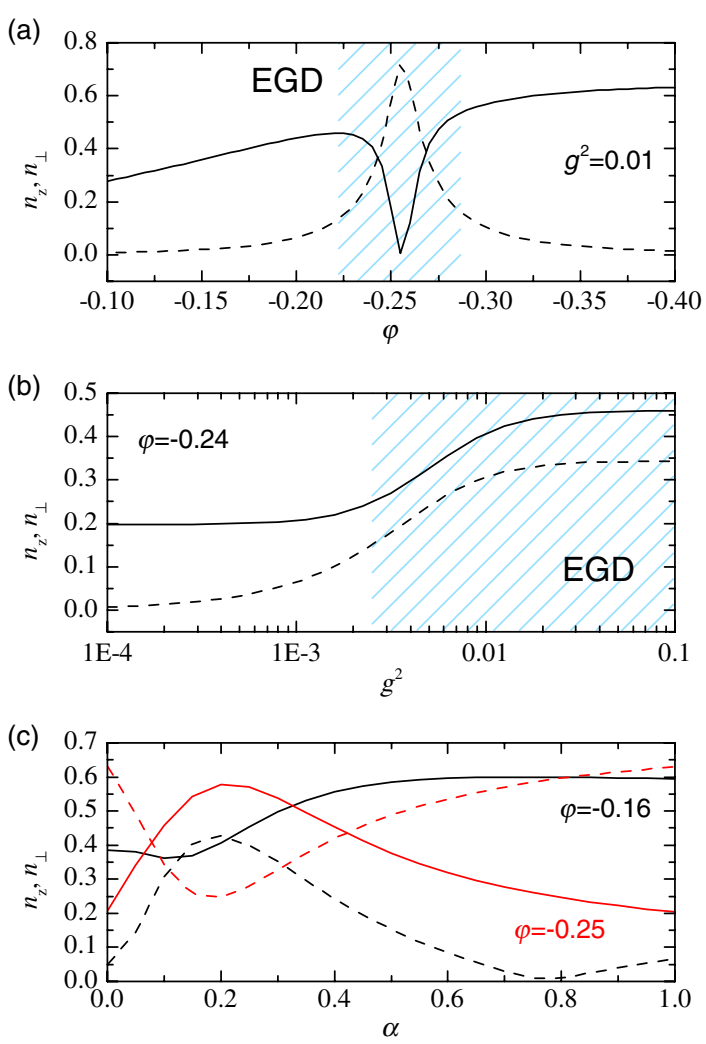

FIG. 2 (color online). SGD to EGD transition. $n_{z}$ (full lines) and $n_{\perp}$ (dashed lines) versus $\varphi$ for $A=\sigma_{z}$ and $g^{2}=0.01$ (a), versus $g^{2}$ for $A=\sigma_{z}$ and $\varphi=-0.24$ (b), and versus $\alpha$ for $\varphi=$ -0.16 (black) and $\varphi=-0.25$ (red) and $g^{2}=0.1$ (c). EGD is attained in the highlighted regions of panels (a), (b), and everywhere in panel (c). The drive parameters are the same as in Fig. 3.

presented in Fig. 2(b). For small values of $g^{2}$, the coherence between Floquet states is completely lost $\left(n_{\perp}=0\right.$, SGD). For larger values of $g^{2}$ (but still in the weak-coupling regime), $n_{\perp}$ takes a finite value (EGD). Notice that as soon as either of the two limits is attained, the steady-state populations do not depend on the exact value of $g^{2}$.

A second distinctive feature of EGD is that the relaxation basis becomes strongly dependent on the type of noise. To demonstrate this, we introduce a family of coupling operators $A(\alpha)=\alpha \sigma_{x}+(1-\alpha) \sigma_{z}$, where $\alpha \in[0,1]$ parametrizes the angle between the reference basis of $H$ and the noise operator. In Fig. 2(c) we then plot $n_{z}$ (solid lines) and $n_{\perp}$ (dashed lines) versus $\alpha$ for two different values of $\varphi$. We set $g^{2}=0.1$, a value ensuring that the EGD limit is attained for both cases. Upon changing $\alpha, \rho_{\text {st }}$ undergoes significant changes. This is in stark contrast to what happens in SGD, where relaxation always takes place in the same (Floquet) basis regardless of the coupling operator.

As $\rho_{\text {st }}$ is determined by a set of algebraic equations, obtained by setting $\dot{\rho}_{\alpha \beta}=0$ in Eq. (1), these features can be analytically addressed. In the SGD limit, we obtain the same results as predicted by a full secular approximation: $\rho_{11}^{\text {st }}=\rho_{11, \mathrm{SGD}}$, where $\rho_{11, \mathrm{SGD}}$ is a constant whose explicit 
(a)

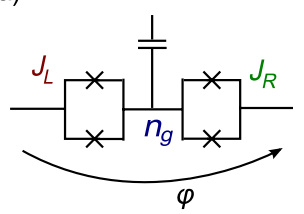

(b)

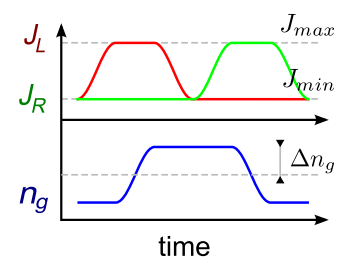

(c)

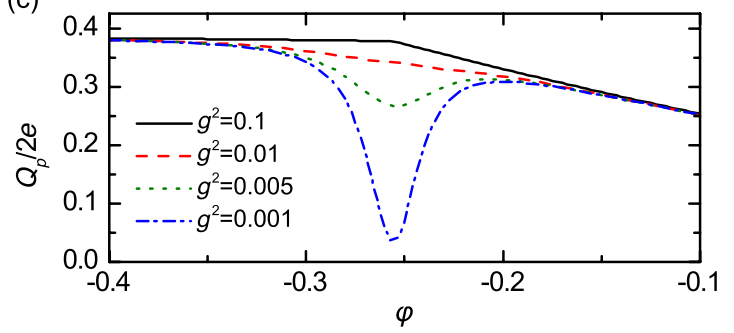

FIG. 3 (color online). Application to the Cooper-pair sluice. (a) Equivalent circuit of the sluice. (b) Time dependence of $J_{L}$, $J_{R}$, and $n_{g}$ during a pumping cycle. (c) Pumped charge $Q_{p}$ versus $\varphi$ for selected values of $g^{2}$. Relevant drive parameters are $\tau=$ $1 \mathrm{~ns}, E_{C}=1 \mathrm{~K}, \Delta n_{g}=0.2, J_{\max }=0.1 E_{C}, J_{\min }=10^{-3} J_{\max }$.

expression is found in literature [13,15], and $\rho_{12}^{\mathrm{st}}=O(\chi)$. By contrast, in the EGD limit, $\rho_{11}^{\text {st }}=\rho_{11 \text {,EGD }}$ and $\rho_{12}^{\text {st }}=$ $\rho_{12, \mathrm{EGD}}+O(1 / \chi)$. The constants $\rho_{11, \mathrm{SGD}}, \rho_{11, \mathrm{EGD}}$ and $\rho_{12, \mathrm{EGD}}$ are defined in Ref. [31].

Altogether, the results of Fig. 2 indicate that in the EGD regime the environment is drastically influencing the dynamics of the system. A measurement of $n_{x, y, z}$ can thus disclose valuable information on both the type of noise and the strength of system-environment coupling.

Observation in a superconducting charge pump.-We now discuss how to observe the transition in a superconducting charge pump, the Cooper-pair sluice [21,22,32]. The sluice, shown schematically in Fig. 3(a), consists of a single superconducting island, coupled to superconducting leads via two superconducting quantum interference devices (SQUIDs). The SQUIDs are operated as Josephson junctions of tunable energies $J_{L, R}(t)$. A gate electrode capacitively coupled to the island provides a third control parameter by inducing a polarization charge $n_{g}(t)$ in units of $2 e$. The device is operated under a constant superconducting phase bias $\varphi_{S}$. In the charging regime $E_{C} \gg J_{L, R}$ ( $E_{C}$ is the charging energy of the island), the dynamics can be reduced to the two lowest-energy charge states $|0\rangle$ and $|1\rangle$ corresponding to zero Cooper pairs and one excess Cooper pair on the island, respectively. The system is then described by a pseudospin Hamiltonian of the form discussed above, with effective field components $B_{x}(t)=$ $\frac{1}{2} J_{+}(t) \cos \frac{\varphi}{2}, B_{y}(t)=\frac{1}{2} J_{-}(t) \sin \frac{\varphi}{2}$, and $B_{z}(t)=E_{C}[1 / 2-$ $\left.n_{g}(t)\right]$, where $J_{ \pm}(t)=J_{L}(t) \pm J_{R}(t)$. Pumping is achieved by steering the three parameters $J_{L}, J_{R}$, and $n_{g}$ in a periodic fashion, as shown in Fig. 3(b).

Starting from the given definitions, it can be shown that the pumping cycle of the sluice is a realization of the loop of Fig. 1(a), with $\varphi=\varphi_{S}$. The quasienergy gap of the sluice can thus be tuned by changing the superconducting phase bias while performing exactly the same pulse sequence. This makes the sluice an excellent candidate to verify our theoretical predictions through a direct measurement of the pumped charge $Q_{p}[32,33]$.

The main source of decoherence in the sluice is charge noise, due to fluctuations in the gate voltage $[7,14]$. We describe it by putting $A=\sigma_{z}, \alpha=1$ and $g=C_{g} / C_{\Sigma}$, where $C_{g}$ and $C_{\Sigma}$ are the gate-to-island capacitance and total island capacitance, respectively.

In Fig. 3(c) we plot $Q_{p}$ as a function of $\varphi$ for different coupling strengths $g^{2}$. For small values of $g^{2}$, corresponding to $\chi \ll 1, Q_{p}$ exhibits a dip around $\varphi_{c}$. The dip appears in coincidence with the weakly avoided crossing in the quasienergy spectrum [Fig. 1(b)], and stems from the mixing of "adiabatic" Floquet states at the crossing [15,34]. As $g^{2}$ is increased, an expanding neighborhood of $\varphi_{c}$ undergoes the transition to EGD, producing an increase in $Q_{p}$. Finally, for large enough values of $g^{2}$ the whole region of mixing is in the EGD regime, and the dip has disappeared. The observation of a finite pumped charge at the avoided quasienergy crossing requires quantum coherence between Floquet states [35]; for this reason, it should be regarded as a direct signature of EGD.

Conclusions.-A driven quantum system interacting with the environment exhibits a richer scenario than an undriven one. This is due to the emergence of an energy scale, the Floquet gap, that can compete with decoherence rates in the vicinity of a quasienergy crossing. This energy can be tuned by choosing the drive parameters. We have identified two dynamical regimes and have given an effective coupling parameter governing the transition between the two. This transition manifests itself in the quasistationary density matrix, in particular, in the revival of coherences between Floquet states. Finally, we have discussed how to observe the transition in a superconducting charge pump. A closely related system that can be considered in a similar spirit is the driven Cooper-pair box $[36,37]$.

While the SGD regime has been intensively studied, the EGD regime is vastly unexplored. Because of its simplicity and applicability to a variety of different systems, the present approach may emerge as a useful tool to study system-environment interactions in open quantum systems.

We would like to thank J. Ankerhold, C. Ciuti, V. Gramich, M. Möttönen, J. Salmilehto, and M. Silveri for useful discussions. This work was supported by the Finnish National Graduate School in Nanoscience, by the European Community's Seventh Framework Programme under Grant Agreement No. 238345 "GEOMDISS," and partially by the Academy of Finland through its Centres of Excellence Program (Project No. 251748). P. S. acknowledges financial support from FIRBFuturo in Ricerca 2012 under Grant No. RBFR1236VV "HybridNanoDev." 
*simone.gasparinetti@aalto.fi

[1] H. P. Breuer and F. Petruccione, The Theory of Open Quantum Systems (Oxford University, Oxford, 2007).

[2] W. H. Zurek, Rev. Mod. Phys. 75, 715 (2003).

[3] M. A. Nielsen and I.L. Chuang, Quantum Computation and Quantum Information (Cambridge University Press, Cambridge, England, 2000).

[4] C. Myatt, B. King, Q. Turchette, C. Sackett, D. Kielpinski, W. Itano, C. Monroe, and D. Wineland, Nature (London) 403, 269 (2000).

[5] D. Kielpinski, V. Meyer, M. A. Rowe, C. A. Sackett, W. M. Itano, C. Monroe, and D. J. Wineland, Science 291, 1013 (2001).

[6] H. Weimer, M. Müller, I. Lesanovsky, P. Zoller, and H. P. Büchler, Nat. Phys. 6, 382 (2010).

[7] P. Solinas, M. Möttönen, J. Salmilehto, and J. P. Pekola, Phys. Rev. B 82, 134517 (2010).

[8] J. T. Barreiro, M. Müller, P. Schindler, D. Nigg, T. Monz, M. Chwalla, M. Hennrich, C. F. Roos, P. Zoller, and R. Blatt, Nature (London) 470, 486 (2011).

[9] S. Diehl, E. Rico, M. A. Baranov, and P. Zoller, Nat. Phys. 7, 971 (2011)

[10] B.-H. Liu, L. Li, Y.-F. Huang, C.-F. Li, G.-C. Guo, E.-M. Laine, H.-P. Breuer, and J. Piilo, Nat. Phys. 7, 931 (2011).

[11] P. Solinas, M. Möttönen, J. Salmilehto, and J. P. Pekola, Phys. Rev. B 85, 024527 (2012).

[12] K. W. Murch, U. Vool, D. Zhou, S. J. Weber, S. M. Girvin, and I. Siddiqi, Phys. Rev. Lett. 109, 183602 (2012).

[13] M. Grifoni and P. Hänggi, Phys. Rep. 304, 229 (1998).

[14] J. P. Pekola, V. Brosco, M. Möttönen, P. Solinas, and A. Shnirman, Phys. Rev. Lett. 105, 030401 (2010).

[15] A. Russomanno, S. Pugnetti, V. Brosco, and R. Fazio, Phys. Rev. B 83, 214508 (2011).

[16] F. Pellegrini, C. Negri, F. Pistolesi, N. Manini, G. E. Santoro, and E. Tosatti, Phys. Rev. Lett. 107, 060401 (2011).

[17] S. Diehl, A. Micheli, A. Kantian, B. Kraus, H. P. Büchler, and P. Zoller, Nat. Phys. 4, 878 (2008).

[18] A. Ferrón, D. Domínguez, and M. J. Sánchez, Phys. Rev. Lett. 109, 237005 (2012).

[19] F. Galve, L. A. Pachón, and D. Zueco, Phys. Rev. Lett. 105, 180501 (2010).

[20] J. Cai, S. Popescu, and H.J. Briegel, Phys. Rev. E 82, 021921 (2010).
[21] A. O. Niskanen, J.P. Pekola, and H. Seppä, Phys. Rev. Lett. 91, 177003 (2003).

[22] A. O. Niskanen, J. M. Kivioja, H. Seppä, and J. P. Pekola, Phys. Rev. B 71, 012513 (2005).

[23] R. Blümel, A. Buchleitner, R. Graham, L. Sirko, U. Smilansky, and H. Walther, Phys. Rev. A 44, 4521 (1991).

[24] K. Blum, Density Matrix Theory and Applications (Springer, New York, 2012), 2nd ed.

[25] T. Dittrich, B. Oelschlägel, and P. Hänggi, Europhys. Lett. 22, 5 (1993).

[26] B. Oelschlägel, T. Dittrich, and P. Hänggi, Acta Phys. Pol. B 24, 845 (1993).

[27] I. Kamleitner and A. Shnirman, Phys. Rev. B 84, 235140 (2011).

[28] S. N. Shevchenko, S. Ashhab, and F. Nori, Phys. Rep. 492 , 1 (2010).

[29] S. Gasparinetti, P. Solinas, and J. P. Pekola, Phys. Rev. Lett. 107, 207002 (2011).

[30] The finite-temperature behavior is qualitatively similar to that reported as long as the condition $k_{B} T \ll\langle\Delta E\rangle$, where $\langle\Delta E\rangle$ is the mean energy gap.

[31] See Supplemental Material at http://link.aps.org/ supplemental/10.1103/PhysRevLett.110.150403 for a formal derivation of the scaling properties of the master equation solution.

[32] M. Möttönen, J. J. Vartiainen, and J. P. Pekola, Phys. Rev. Lett. 100, 177201 (2008)

[33] S. Gasparinetti, P. Solinas, Y. Yoon, and J. P. Pekola, Phys. Rev. B 86, 060502(R) (2012).

[34] D. W. Hone, R. Ketzmerick, and W. Kohn, Phys. Rev. E 79, 051129 (2009).

[35] As shown in Ref. [15], the charge carried by a Floquet state is proportional to the derivative of the corresponding quasienergy with respect to the phase bias $\varphi$. At a symmetrically avoided quasienergy crossing, this implies that the transferred charge vanishes for any incoherent superposition of the two Floquet states.

[36] C. M. Wilson, T. Duty, F. Persson, M. Sandberg, G. Johansson, and P. Delsing, Phys. Rev. Lett. 98, 257003 (2007).

[37] C. M. Wilson, G. Johansson, T. Duty, F. Persson, M. Sandberg, and P. Delsing, Phys. Rev. B 81, 024520 (2010). 